\title{
CONSTRUCCIÓN DEL PERSONAJE ADOLESCENTE EN LA FICCIÓN SERIADA EUROPEA. LAS SERIES ORIGINALES DE NETFLIX COMO CASO DE ESTUDIO
}

\section{Characteristics of the Teenager in European Serial Fiction. Netflix Originals as a Case Study}

\section{Ldo. Daniel FERRERA}

Doctorando. Universidad Carlos III de Madrid, España

E-mail: daniel.ferrera@alumnos.uc3m.es

(iD) https://orcid.org/0000-0002-7029-2112

Fecha de recepción del artículo: 09/05/2020

Fecha de aceptación definitiva: 16/10/2020

\begin{abstract}
RESUMEN
El presente artículo analiza la construcción de la identidad adolescente en la ficción seriada europea a través del análisis de personajes en 23 series originales de Netflix utilizando una metodología que aúna elementos cuantitativos y analítico-descriptivos. Para establecer las diferencias y similitudes entre los personajes adolescentes de las distintas series europeas, se han clasificado un total de 132 personajes en torno a 18 variables de análisis, centrando la atención tanto en cuestiones de representación como en aspectos de la trama, destacando la importancia del grupo de pares para el personaje adolescente, así como la existencia de un comportamiento que, especialmente en lo relativo a la sexualidad, resulta incongruente con la edad que representa.
\end{abstract}

Palabras clave: estudios televisivos; ficción televisiva; ficción televisiva europea; televisión a la carta; adolescentes; Netflix.

\begin{abstract}
This paper analyses the construction of the teenage character in European serial fiction through the analysis of 23 Net-flix Originals with a methodology that combines quantitative and analytical-descriptive elements. To establish the dif-ferences and parallels between the teenage characters of the different European series, a total of 132 characters have been classified around 18 variables of analysis, focusing on issues of representation and aspects of the storyline, emphasizing the importance of the peer group for the teenage character, as well as the existence of a behavior that, sometimes, is in-consistent with the age it portrays.
\end{abstract}

Key words: television studies; television fiction; European television fiction; on-demand television; teenagers; Netflix. 


\section{Introducción}

El 28 de abril de 2017, Netflix estrenó la primera de sus series originales de producción española, Las chicas del cable (2017-2020), tan solo año y medio después de iniciar operaciones en España (Cascajosa Virino, 2018, p. 58). Adelantándose de esta forma cinco meses a Movistar+, que en septiembre de ese mismo año estrenaría Velvet Colección (2017-2019), y cerca de tres años a Amazon Prime Video, que hasta 2020 no presentaría una serie original de producción española con Caronte (2020-), la estrategia utilizada por Netflix sería la misma que utilizarían después el resto de plataformas de streaming en el contexto de producción español: seguir una tendencia continuista con respecto a la ficción televisiva tradicional, aprovechando la experiencia de profesionales de reconocida trayectoria y aliándose con productoras de amplio recorrido. De esta forma, para su primera serie española, Netflix contó con Bambú Producciones y con Ramón Campos y Gema R. Neira como creadores. Tres años después de esta primera producción, y cerca de cuatro años y medio después de su llegada a territorio español, el número de series originales de Netflix accesibles desde España el 28 de abril de 2020, englobadas en la plataforma bajo el código 839338, asciende a un total de 480 (teniendo en cuenta únicamente las producciones de ficción), de las cuáles son españolas, además de la ya mencionada Las chicas del cable, Paquita Salas (2016-) y La casa de papel (2017-), adquiridas y continuadas tras su paso por canales del grupo Atresmedia, Élite (2018-), Alta mar (2019), Hache (2019-), El vecino (2020-) y la serie antológica Criminal: España (2019-), creada por George Kay y Jim Field Smith y con versiones para Reino Unido, Francia y Alemania. Por tanto, únicamente el 1,66 \% de las producciones seriadas originales de Netflix accesibles desde España entre marzo y abril de 2020 son de producción nacional. Calificadas para mayores de 16 años salvo Paquita Salas, Criminal: España y El vecino, para mayores de 13, todas estas series están consideradas como producciones aptas para el público adolescente, si bien su target engloba a una audiencia más amplia. Esta clasificación por edades refleja, en el conjunto de las 480 producciones seriadas disponibles en España, una preeminencia por la ficción dirigida a adolescentes, lo cual enlaza con el modelo de negocio de Netflix, basado en "suscripciones económicas no muy elevadas, asequibles para el público joven al que se dirigen" (Ojer y Capapé, 2012, p. 201). Con un 8,54\% de las ficciones originales dirigida a todos los públicos y un 12,5\% para mayores de 7 años, el grueso de las series originales de Netflix se clasifica en torno a las categorías de mayores de 16 (45,62\%) y mayores de 13 (31,66\%), sumando entre ambas el 77,29 $\%$ del total, y limitándose el número de producciones no consideradas aptas para menores de 18 al 1,66\%.

Estos datos, elaborados a partir del catálogo de la plataforma, posicionan a Netflix como un servicio de difusión para el que el público adolescente resulta clave, al menos desde el punto de vista de la producción original. Esta preeminencia de series para mayores de 16 años (y, en menor medida, de 13) enlaza con una mayoría de producciones estadounidenses, un $37,08 \%$ del total, que alcanza el 41,87\% si se añaden las coproducciones entre Estados Unidos y otros países como Canadá, Reino Unido, Japón o Corea del Sur. Por tanto, en el contexto de recepción español, el catálogo de Netflix ofrece en mayor medida ficciones americanas para el público adolescente. Esta homogeneización en los contenidos, que enlaza con la de las especificaciones técnicas requeridas a las producciones originales para formar parte del catálogo de Netflix, encuentra su justificación en la propia naturaleza transnacional 
de la plataforma y su audiencia global (Jenner, 2018, pp. 241-257). Si bien el calificativo de original responde a diversos criterios (series encargadas y producidas por la propia Netflix, coproducidas con alguna otra cadena, continuación de series de otras net Works o aquellas para las que tiene los derechos de distribución internacional), la serie de ficción se constituye en el contexto de las producciones originales de Netflix como un producto manufacturado de forma homogénea en un ejercicio de imperialismo cultural, tendencia iniciada en las televisiones de cobertura nacional, como en el caso español, que «importaron casi todas sus series extranjeras de las net Works estadounidenses» así como "sus tendencias de programación» (Fernández-Ramírez y McGowan, 2020, p. 550). De esta forma, y mientras que Mikos (2019, p. 7) apunta la necesidad de entender la televisión dentro del contexto político, legal y económico de cada país, teniendo en cuenta que lo transnacional queda supeditado a las regulaciones de carácter nacional, la naturaleza misma de Netflix, que se construye en torno al concepto de lo "global» (Lobato y Lotz, 2020, p. 133), posiciona a la plataforma como un medio de distribución transnacional en el que, si bien destaca la representación de la diversidad tanto en términos raciales como de género, esta queda definida desde un punto de vista norteamericano (Jenner, 2018, p. 219).

La presente investigación está estructurada en torno a dos hipótesis de partida. La primera es que esta homogenización detectada en el catálogo de producciones seriadas originales puede aplicarse también a su contenido; de esta forma, independientemente del país de origen, la construcción de los personajes y las dinámicas establecidas entre los mismos atenderán a patrones similares. Centrando la mirada en las producciones europeas, y debido a la primacía de las series categorizadas para un público de más de 16 y 13 años, en los personajes adolescentes protagonistas, la segunda de las hipótesis que impulsan la investigación es que esta tipología de personajes se construye en función de la representación de roles y estereotipos, jugando para ello un papel muy importante su grupo de pares (Raya, Sánchez-Labella y Durán, 2018; Forni, 2020, pp. 303-304), desenvolviéndose en determinadas situaciones de una forma que no se adecúa a los comportamientos propios de personas en su rango de edad (Davies y Dickinson, 2004, p. 136), especialmente en lo relativo a las relaciones de carácter sexual. De esta forma, los objetivos principales son, por un lado, analizar el grado de protagonismo de los personajes adolescentes en la ficción seriada europea, es decir, qué porcentaje de series originales acreditan a los intérpretes de este tipo de personajes dentro del reparto regular y si existen diferencias significativas entre los distintos países; en segundo lugar, definir los rasgos comunes en la construcción del perfil del personaje adolescente para establecer diferencias y/o similitudes; y, por último, analizar las relaciones establecidas entre esta tipología de personajes y su grupo de pares para establecer si sus pautas de comportamiento se ajustan o no a las esperables en personas de su rango de edad.

La metodología empleada, que mezcla elementos cuantitativos con un análisis cualitativo-descriptivo, fundamenta la elección del corpus en una base de datos elaborada a partir de las producciones seriadas disponibles en el catálogo de originales de Netflix, consultado desde España entre el 13 de marzo de 2020 (día del estreno de la tercera temporada de Élite, serie de adolescentes por antonomasia entre las producciones españolas originales de la plataforma) y el 28 de abril del mismo año (día en el que se cumplen tres años desde la primera emisión de una serie original de producción española en Netflix); dicha base de datos, organizada en torno a 12 variables de análisis (título en español, título original, país, género, año de emisión, 
creador/es, cadena original, número de temporadas, número de episodios, duración y calificación por edades), servirá para realizar una primera aproximación al objeto de estudio y establecer, asimismo, una serie de porcentajes mediante los que comparar cuantitativamente el grado de relevancia y la construcción de los personajes protagónicos adolescentes en los distintos países europeos. Posteriormente, y una vez establecidas las series conformantes del corpus, se seleccionarán, de acuerdo con los títulos de crédito de estas, aquellas con protagonistas adolescentes, clasificando a los mismos de acuerdo a una ficha de análisis elaborada expresamente para la presente investigación.

\section{Adolescencia y ficción televisiva. Contextualización}

Si bien la importancia de las series de televisión como uno de los productos culturales con mayor relevancia en la actualidad es innegable, el proceso de legitimación de las mismas se ha visto claramente influido por las comparaciones con otros medios, como el cine o la literatura, consolidando además, desde el aparato crítico, un canon del que quedan fuera muchas producciones seriadas de éxito englobadas bajo el término de "guilty pleasures» en un ejercicio de «degradación de la experiencia espectatorial y un reflejo de una jerarquía cultural que pretende no solo categorizar a las series sino también a sus espectadores» (Cascajosa Virino, 2016, p. 175). Esta clasificación establece, desde una óptica norteamericana, un sistema de valor en el que la crítica se constituye como elemento esencial para la supervivencia de un determinado programa (Cascajosa Virino, 2016, p. 152), especialmente en un contexto de multiplicidad de canales y plataformas (Mikos, 2019, p. 7) que deriva, en un proceso iniciado en el año 2005 (Roel, 2020), en una tendencia hacia una televisión personal individualizada (Álvarez Monzoncillo, 2011, pp. 83-101). Las cadenas televisivas de ámbito europeo, que incluyen en sus parrillas una gran cantidad de ficción estadounidense (Meier, 2016, p. 50), encuentran para sus respectivos ámbitos nacionales un proceso crítico y jerarquizador similar, siendo posiblemente el caso español el más extremo, en el que durante la pasada década era habitual encontrar comparativas en términos de calidad entre las series nacionales y las estadounidenses, siempre en detrimento de las primeras (Cascajosa Virino, 2016, pp. 213-249).

La implantación de las plataformas de streaming, tanto en España como a nivel mundial, ha permitido un mayor grado de internalización en el sector que, no obstante, no ha sido óbice para que el contenido norteamericano continúe primando sobre el resto, como demuestra el 37,08 \% de series originales estadounidenses de Netflix accesibles a comienzos del mes de abril desde España (41,87 \% contando las coproducciones) frente al 1,66 \% de producciones nacionales. No obstante, los tres títulos más vistos en Netflix fuera de Estados Unidos la semana del 23 al 29 de marzo fueron españoles: una serie original, Élite, una producción externa de la que posee los derechos de distribución internacional, Toy Boy (Antena 3, 2019), y una película, El hoyo (Gaztelu-Urrutia, 2020) (Border, 14 de abril de 2020). De esta forma, si bien la internalización de contenidos a través de las plataformas de streaming prioriza los producidos en Estados Unidos, la audiencia dispone de un amplio catálogo de ficciones de diversas nacionalidades entre las que elegir, no teniendo por qué coincidir sus elecciones con los productos hegemónicos. Pero eimplica esta capacidad de elección la posibilidad de acceder a producciones de marcado carácter nacional o, por el contrario, se encuentra 
en todas ellas, en mayor o menor grado, una clara intención homogeneizadora en sus formas y contenidos? Si las especificaciones técnicas requeridas para formar parte del catálogo de contenido original de Netflix equiparan a todas estas producciones en términos visuales y la intención de distribuir internacionalmente el texto audiovisual condiciona su producción (Ahn, 2013, p. 364), ¿existe alguna característica más allá del idioma original o la ubicación de sus tramas que identifique a las series como pertenecientes a una determinada nacionalidad o prevalece la naturaleza transnacional de la propia plataforma?

Uno de los cambios fundamentales derivados de la difusión de series en streaming con respecto a los canales de televisión tradicionales es, más allá del cambio en el modelo de negocio en el que la publicidad es sustituida por el pago de cuotas, cómo varía la recepción del contenido, desligándolo de una parrilla que lo fija a un horario determinado (Herbert, Lotz y Marshall, 2019, pp. 361-364). Eliminando así la simultaneidad del visionado, y permitiendo al espectador fijar la cadencia entre episodios, la experiencia socializadora del consumo televisivo se amplifica a través del uso de las redes sociales para una audiencia global que no puede interpretarse en términos de territorio. Por tanto, y para que dicha experiencia socializadora pueda tener lugar, las ficciones necesitan estructurarse en torno a unos códigos comunes, unos valores y/o experiencias compartidos entre los miembros de una audiencia dispersa que no comparten necesariamente una misma nacionalidad. Más allá del grupo de pares del entorno más inmediato, el espectador de la ficción seriada distribuida en streaming en un ámbito global encuentra a través de Internet y las redes sociales un grupo de iguales transnacional. La audiencia adolescente, para la que el entorno mediático juega un papel crucial en cuanto a la construcción de su identidad (Belmonte y Guillamón, 2008), necesita por tanto ser entendida transculturalmente (Medrano, Martínez de Morentín y Apodaca, 2015) dentro de un contexto multimedia (Calvo González y Saavedra Álvarez, 2020, p. 54) en el que las nuevas tecnologías proporcionan al adolescente un entorno multipantallas del que reciben estímulos continuos (Fedele y García-Muñoz, 2010, pp. 51-53). De esta forma, «el visionado audiovisual, especialmente el de las audiencias más jóvenes, se está redirigiendo a nuevas pantallas» (Capapé, 2020, p. 453) y el espectador adolescente aprovecha las nuevas tecnologías para desempeñar un rol más activo, excediendo las fronteras de la propia televisión y generando un nuevo tipo de espectador (nativo digital) en un contexto en el que una adolescencia mediatizada "lo inunda todo y se contamina de elementos propios de la adultez a través de unos productos que cuentan con las características de producción, distribución y exhibición propias de adultos pero protagonizados por niños y adolescentes» (Guarinos-Galán, 2009, p. 205). Los mecanismos de identificación de la audiencia adolescente con los personajes, mediante procesos de empatía cognitiva y, en menor medida, conceptual (Medrano, Martínez de Moratín y Apodaca, 2015, p. 317), juegan un enorme papel en la construcción de la identidad del adolescente, a través del impacto de los rasgos considerados atractivos entre los personajes regulares (Hoffner y Buchanan, 2005, pp. 345-347); por tanto, un catálogo de ficción construido en términos transnacionales con una perspectiva unitaria contribuiría a la homogenización de una audiencia en el ámbito global, de forma que las nuevas generaciones encontrarían más puntos en común con personas de su edad, independientemente de su contexto geopolítico, a pesar de la posible existencia de una mayor afinidad con personajes de su misma nacionalidad (Lacalle, 2015, p. 250), que individuos coetáneos de generaciones anteriores. 
Los personajes adolescentes representados en la ficción seriada se desarrollan a través de tramas y conflictos ligados a la interacción con su grupo de pares, con temáticas centradas en el amor y la amistad (García-Muñoz y Fedele, 2005), jugando un papel crucial la sexualidad (Masanet, Medina-Bravo y Ferrés, 2012). Y es ese contexto, en el que "se desenvuelven e interactúan con otras personas o personajes, encontrándose todas ellas inmersas en un escenario de acción donde se desarrolla una trama que toma sus contenidos de la realidad o de la ficción» (Bermejo, 2012, pp. 31-32), el que resulta decisivo en la asimilación cognitiva de las dinámicas sociales por parte de la audiencia adolescente. La denominada "teen tv" se constituye como el espacio ideal para la negociación de significados sociales, culturales y políticos (Ross y Stein, 2008, p. 1), resultando complejo su categorización como la de todo género televisivo (Creeber, 2004, pp. 1-3), pero siendo tradicionalmente asociada tanto a la audiencia de la misma como a su contenido (Ross y Stein, 2008, pp. 4-5). La dificultad a la hora de definir los parámetros de un determinado género, derivada de la naturaleza misma del medio televisivo y su adaptación de formatos desde otros medios (Neale, 2004, p. 3), se multiplica en el entorno transnacional, donde la audiencia de las plataformas de streaming queda difuminada en términos geopolíticos, y la convergencia mediática que posibilita la aparición de una audiencia activa (Jenkins, 2008, p. 14) genera un nuevo contexto de distribución y recepción en el que el adolescente, como nativo digital, queda posicionado en un lugar privilegiado: en un entorno en el que para los estudios de audiencia el cambio tecnológico y la aparición de nuevas formas de visionado como el denominado «binge-watching» resulta en la necesidad de redefinir los parámetros de aproximación metodológica al objeto de estudio (Graemer, 2019), las habilidades técnicas del espectador adolescente se constituyen como el elemento indispensable para la existencia del grupo de pares transcultural. La ficción seriada de adolescentes, y para adolescentes, distribuida a través de plataformas de streaming de cobertura global, resulta de esta forma especialmente relevante a la hora de analizar posibles similitudes en cuanto a la construcción y representación de los personajes en producciones de distintas nacionalidades. La existencia de estas favorecería la construcción, a nivel transnacional, de generaciones inter pares con una serie de características comunes derivadas de la perspectiva dominante en la representación de las ficciones audiovisuales, es decir, el punto de vista de la ficción norteamericana, especialmente en el caso de las audiencias adolescentes, en pleno desarrollo identitario. El presente artículo se centra en el análisis de la posible existencia de dichas similitudes, dejando a futuras investigaciones el estudio de las implicaciones que estas podrían tener en una audiencia transnacional.

\section{Ficción seriada europea. Aproximación y propuesta metodológica}

El análisis de las producciones originales accesibles en el catálogo de Netflix desde España entre mediados de marzo y principios de abril de 2020, en el que se contabilizan un total de 480 ficciones seriadas, revela la existencia de 91 series con origen exclusivamente europeo (excluyendo 9 coproducciones entre países europeos y Australia, Canadá, Estados Unidos, México y Nuevo Zelanda), lo que supone un $18,95 \%$ del total, equivalente a la mitad de las producciones estadounidenses. Del total de ficción europea, el 39,56 \% se corresponde con series producidas en Reino Unido, quedando Francia (14,28 \%) y España (8,79 \%) en segundo y tercer lugar. Por 
el contrario, en términos globales, las producciones francesas representan el 2,7 \% y las españolas el 1,66 \%, y Reino Unido se sitúa en cuarto lugar, con un 7,5\%, por detrás de Estados Unidos (37,08 \%), Japón (10,83 \%) y Corea del Sur (8,95 \%).

Como ya se ha mencionado, los requisitos para formar parte del catálogo de originales de Netflix responden a diversos criterios entre los que se incluyen, además de series en cuya producción ha participado, aquellas para las que posee los derechos de distribución. De esta forma, una serie como la británica Derry Girls (Channel 4, 2018-) aparece englobada dentro del término original en el catálogo consultado desde España, pero su concepción como original varía en función del país desde el que se acceda a la plataforma. Por tanto, y en aras de eliminar posibles discrepancias, para el presente análisis se ha optado por excluir todas aquellas series adquiridas por Netflix para su distribución tras su paso por los canales de sus respectivos países de origen, pero sí incluyendo aquellas, como La casa de papel, que han sido continuadas por la plataforma (teniendo en cuenta para su análisis únicamente las temporadas producidas para su emisión exclusiva en Netflix) o las que, como Drácula (BBC One, 2020), fueron estrenadas en canales tradicionales pero coproducidas por Netflix con la intención, desde el momento de su concepción, de ser la encargada de su distribución internacional. De esta forma, el 18,95 \% de producciones europeas queda reducido al $10 \%$, con un total de 48 títulos, continuando Reino Unido como líder, con 14 títulos y el 29,16\% del total de los países europeos, seguido de los 8 de España y Francia (16,66 \%), pero únicamente con el 2,91 \% y el 1,66 \% del cómputo global.

Realizado este primer filtro, para establecer el corpus de análisis definitivo se han seleccionado aquellas de estas 48 series que cuentan entre sus protagonistas (cuyos intérpretes aparecen acreditados como parte del elenco principal regular de la serie) a personajes adolescentes. Para la presente investigación se considerará adolescente, si bien resulta necesario tener en cuenta la disparidad de criterios existentes y la problemática derivada de la "contradicción entre el adelanto social de la adolescencia y el retraso de independencia y comportamientos sociales y psicológicos» (Guarinos-Galán, 2009, p. 205), a todo personaje cuya edad quede comprendida entre los trece y los diecinueve años, es decir, aquellas que en inglés contienen el sufijo «teen", del que surge el término "teenager» en el contexto estadounidense de 1944 para referirse a "la juventud como mercado concreto y de masas» (Savage, 2018 p. 543). De esta forma, el corpus de análisis queda compuesto por un total de 23 producciones: las alemanas Cómo vender drogas online (a toda pastilla) (How to Sell Drugs Online (Fast), 2019-) y Dark (2017-2020), las británicas A rienda suelta (Free Rein, 2017-2019), No bables con extraños (The Stranger, 2020-), Safe (2018), Sex Education (2019-), The Crown (2016-) y The Innocents (2018), la danesa The Rain (2018-), las españolas Élite y Las chicas del cable, las francesas Mortal (Mortel, 2019-), Osmosis (2019) y Vampiros (Vampires, 2020-), la islandesa Case (2015), las italianas Baby (2018-), Luna Nera (2020-) y Suburra (Suburra - La serie, 2017-), la noruega Navidad en casa (Hjem til jul, 2019), la neerlandesa Ares (2020-), las suecas Arenas movedizas (Störst av allt, 2019) y Kalifat (2020) y la coproducción noruego-danesa Ragnarok (Ragnarök, 2020-). Con una presencia desigual en las diferentes ficciones, y el 69,56\% de las producciones dirigidas a mayores de 16 años (salvo seis títulos para mayores de 13, Navidad en casa, No hables con extraños, Osmosis, The Crown, The Innocents y The Rain, y A rienda suelta, único de los 48 títulos europeos calificado para mayores de 7), el número de personajes adolescentes analizados asciende a un total de 132. 
Para su estudio se ha procedido a la elaboración de una ficha de análisis basada en las proposiciones de Casetti y de Chio (2007, pp. 177-188) relativas a los personajes, teniendo en cuenta el esquema actancial de Greimas (1987) y las tipologías establecidas por Propp (2001), tomando además elementos de la propuesta metodológica ideada por Galán Fajardo (2006). De esta forma, los personajes serán categorizados en una base de datos en torno a 18 variables para su análisis, elaborada tras el visionado de todos los episodios de las 23 series propuestas. En primer lugar, se indicará el título de la serie a la que pertenecen, así como la nacionalidad y el género de esta. Posteriormente, y antes de centrarse en el personaje propiamente dicho, se especificará la época en la que se desarrolla la trama, distinguiendo entre la actualidad, una época histórica o el futuro. A partir de la quinta variable, y hasta la decimoprimera, se recogerán los datos relativos al nombre del personaje, su intérprete, la edad o el rango de edad en el que se encuentra (dependiendo de los años que abarque la trama), el sexo, la identidad de género, la nacionalidad y la raza. Tras estas categorías, fundamentales para establecer relaciones y porcentajes entre los distintos personajes analizados, las variables restantes se centrarán en las características susceptibles de análisis: representación icónica (atractiva, promedio, poco atractiva; en función no de la opinión propia sino la del entorno del personaje), rasgo distintivo (en caso de poseerlo, como puede ser algún tipo de marca o la utilización de un determinado accesorio característico con relevancia para el desarrollo del personaje o del argumento), personalidad (introvertida o extrovertida), objetivos, y su análisis como persona, como rol y como actante. Además de estas 18 variables, se incluye un breve resumen sobre cada personaje, centrado en su recorrido y evolución a lo largo de la trama. De esta forma, las variables de análisis referidas al personaje propiamente dicho servirán para, por un lado, establecer la homogeneización en su construcción (entre la quinta y la decimoprimera, con relación a la primera de las hipótesis planteadas) y analizar la forma en la que se desenvuelve con su grupo de pares, de acuerdo con la representación de roles y estereotipos (a partir de la decimosegunda variable y conforme a la segunda hipótesis de partida).

\section{El perfil del personaje adolescente. Resultados}

Una vez establecido que únicamente 23 de las producciones europeas cuentan con personajes adolescentes acreditados dentro de su elenco regular principal, el visionado de los 305 episodios de estas ficciones seriadas revela un total de 132 personajes que, categorizados en torno a las variables descritas, arrojan una serie de resultados que se detallan a continuación.

En primer lugar, no se observa una preeminencia de personajes de un sexo sobre los del otro, siendo los femeninos 70 y los masculinos 62, pero sí una mayoría de heterosexuales, con un $81,06 \%$ del total, siendo el porcentaje de personajes heterosexuales femeninos superior al de los masculinos, con un 88,57 \% de los primeros frente a un $72,58 \%$ de los segundos. Con un $4,54 \%$ de personajes bisexuales y un $5,3 \%$ cuya sexualidad no queda definida en las tramas desarrollados en las distintas series, los personajes homosexuales representan un 8,33\% del total (10 masculinos frente a 1 femenino), destacando además la existencia de un único personaje autocalificado como pansexual (Ola Nyman en Sex Education). Por el contrario, con respecto a la nacionalidad de los personajes existe menor representación de variedad, única- 
mente con un 3,78 \% de extranjeros. Este hecho no es óbice para que no exista una muestra representativa de diversidad racial (siempre con un predominio de personajes caucásicos, que suponen el 74,24 \% del total), ya que el 88,23\% de los personajes no caucásicos son adolescentes descendientes de inmigrantes y nacidos en el país en el que se desarrollan las tramas de sus respectivas series. De esta forma, la generación de personajes adolescentes encarnada en la ficción europea actual representa la multiculturalidad fruto del contexto migratorio global, en el que el localismo de cada producción queda patente a través de las distintas nacionalidades de los respectivos progenitores, como los padres marroquíes de los hermanos Shanaa en la española Élite, el padre libanés de Damiano Younes en la italiana Baby o la ascendencia india de Anwar y Olivia en la británica Sex Education.

Con personajes mayoritariamente multifacéticos y alejados de la unidimensionalidad (en un 74,24\% del total) y el predominio de personalidades extrovertidas $(74,24$ $\%$, no observándose distinciones entre masculinos y femeninos, únicamente el 16,66 \% está construido en función de objetivos que no guardan relación con encajar en su grupo de pares, fundamentalmente en tramas históricas, de fantasía o ciencia ficción en las que la motivación principal del personaje se encuentra estrechamente ligada al propio contexto del relato en el que se desenvuelven, siendo la propia supervivencia el desencadenante fundamental de sus acciones en la mayoría de los casos.

En relación con el número de personajes presentados en función de la nacionalidad de las series, Reino Unido encabeza el listado, con 40 personajes y un 30,3\% del total, seguido de España e Italia, con 17 (12,87 \%) y Alemania con 16 (12,12 \%). En cambio, si se tiene en cuenta la media de personajes adolescentes en función del número de ficciones, el orden varía sustancialmente, quedando como líder la ficción española con 8,5 adolescentes de media, seguida de la alemana con 8 y la británica con 6,66 (los 9 personajes de la coproducción noruego-danesa la situarían en cabeza, pero no se les ha otorgado el mismo valor al pertenecer a una serie de doble nacionalidad, dividiendo por tanto el resultado entre Noruega y Dinamarca y sumando 4,5 personajes a cada una). De esta forma es Élite, con 16 personajes adolescentes protagonistas, la ficción europea con mayor representación en esta franja de edad, seguida de los 14 de la británica Sex Education y los 10 de la alemana Dark.

Establecidos estos primeros parámetros, y teniendo en cuenta las hipótesis sobre las que se estructura la presente investigación, uno de los aspectos fundamentales detectados en la composición de los personajes adolescentes de las 23 ficciones analizadas tiene que ver con la homogenización en cuanto a la representación de estos, tanto icónicamente como con respecto a sus pautas de comportamiento. Con respecto a la clasificación de los personajes de acuerdo con su representación icónica, predominan aquellos considerados atractivos por su grupo de pares (un 66,66 \%) frente a aquellos considerados poco atractivos (solamente el 5,3\%). Pero en este caso sí se observan diferencias entre mujeres y varones, con una mayor representación de personajes atractivos entre las primeras que entre los segundos. De esta forma, mientras que los adolescentes varones quedan agrupados en un 58,06\% atractivos, un $33,87 \%$ promedio y un $8,06 \%$ poco atractivos, ellas quedan clasificadas con $66,66 \%$, $28,03 \%$ y $1,51 \%$ respectivamente, hecho que enlaza con la concepción de estos como sujeto u objeto, destacando en el caso de los personajes femeninos la necesidad de auto posicionarse como sujetos en un contexto en el que elementos externos tratan de situarlos en la categoría de objeto. 
A la hora de analizar los comportamientos de los distintos personajes, resulta necesario, en primer lugar, tener en cuenta las ficciones en las que se desarrollan los mismos, tanto desde el punto de vista de la trama como en lo tocante a la audiencia a la que se dirigen. Mientras que $A$ rienda suelta, serie dirigida al público más joven, calificada para mayores de 7 años, se desarrolla mayoritariamente a través de episodios auto conclusivos en los que priman los valores del compañerismo y la amistad, con personajes estructurados en torno a la consecución de un objetivo claramente ligado al desarrollo del arco argumental principal de cada temporada, la complejidad narrativa de series como Dark, desarrollada en seis épocas distintas (entre 1921 y 2052), sirve para mostrar la evolución de los personajes desde la infancia hasta la madurez y/o la vejez, permitiéndoles además saltar entre épocas y distintas realidades y otorgando así un mayor grado de profundidad a los personajes. De esta forma, tanto la trama desarrollada como el público al que van dirigidas cada una de estas 23 series influye en la construcción de los personajes adolescentes, destacando especialmente en el contexto de la presente investigación la británica $A$ rienda suelta, en la que la diversidad queda representada únicamente en términos raciales, con personajes enmarcados dentro del centro heteronormativo, y unas tramas afectivas que se centran en mostrar lazos familiares y de amistad. Por otra parte, tanto en Dark, Osmosis y The Rain, con tramas de ciencia ficción, como en Luna Nera, serie de fantasía ambientada en el siglo xviI, y en las ficciones de corte histórico Las chicas del cable (con una quinta temporada ambientada en la guerra civil española) y The Crown (protagonizada por la familia real británica), los personajes adolescentes, con mayor o menor grado de protagonismo, se encuentran profundamente vinculados y condicionados por el entorno en el que se desenvuelven, entroncándose de esta forma sus objetivos con los desencadenantes de sus respectivas tramas (los viajes en el tiempo en Dark, la fusión biotecnológica en Osmosis, la supervivencia en el escenario postapocalíptico en The Rain, la persecución de brujas en Luna Nera, la guerra en Las chicas del cable y las obligaciones derivadas de pertenecer a la realeza en The Crown). Pero todas tienen en común con el resto de las ficciones seriadas analizadas la búsqueda de una identidad propia por parte del personaje adolescente, así como su necesidad de encontrar pareja.

En cuanto a su dimensión sociológica, destaca especialmente que los conflictos externos de cada personaje suelen ser asumidos por los mismos de forma individual para finalmente confiar en su grupo de pares, pero se mantienen fuera del ámbito de actuación de los progenitores que, en la mayoría de las ocasiones, contribuyen a crearlos o amplificarlos en lugar de a su resolución. De esta forma, los comportamientos de los adolescentes se sitúan en una esfera de actuación más responsable y resolutiva que la de los adultos de los que en un principio tendrían que depender. Tomando como hilo conductor la española Élite, debido a que es la serie que más personajes adolescentes presenta, se observan similitudes en las ficciones que podrían clasificarse bajo el término de drama juvenil, ya sea en aquellas que incluyen elementos fantásticos, como Mortal, Vampiros, The Innocents o Ragnarok, o en las que se estructuran en torno a tramas más realistas, como Baby, Cómo vender drogas online (a toda pastilla), Arenas movedizas o Sex Education. Volviendo a Élite, y a las relaciones paternofiliales, se observa una tendencia del progenitor a generar gran parte de los problemas que afectan al personaje adolescente: desde el derrumbe de una escuela de secundaria ocasionado por los intereses económicos de la empresa del padre de los Nunier Osuna, pasando por la incapacidad de la madre de los García Domínguez para mantener a su familia, hasta la obligación de Carla de emparejarse con Yeray en 
contra de su voluntad para que su padre pueda cerrar un trato de negocios con este o la introducción de Rebeka en el mundo del narcotráfico tras el encarcelamiento de su madre y la necesidad de continuar pagando la matrícula del instituto privado al que asisten todos los protagonistas. Las tramas que se inician debido a las acciones de los progenitores sirven para que el personaje adolescente desarrolle un viaje de descubrimiento y enriquecimiento que lo lleva a tomar decisiones necesarias para la resolución del conflicto: Valerio y Rebeka, tras ver cómo el consumo de drogas lleva a Carla a una situación crítica, deciden dejar el negocio llegando Rebeka, en el último episodio de la tercera temporada, a dar un ultimátum a su madre para que cese en sus actividades ilegales; Yeray, por su parte, una vez descubierta la falta de interés por parte de Carla, se alía con ella ofreciendo a su padre el dinero que necesita para salvar la empresa a cambio de que esta pase a ser titularidad de la hija. De esta forma, personajes que alcanzan la mayoría de edad al finalizar el relato (la trama iniciada en el primer episodio de Élite concluye en el final de la tercera temporada), quedan posicionados como jóvenes adultos que han sabido lidiar con una serie de obstáculos y dificultades que, en muchas ocasiones, provenían de sus propios padres.

Líneas argumentales similares se encuentran en el resto de dramas juveniles analizados, por ejemplo en Baby, con Chiara Altieri , hija de un matrimonio en la que el hombre es infiel con el consentimiento de la mujer, que tiene problemas de alcoholismo, y Ludovica Storti, a cargo de una madre que gasta su dinero en contentar a su joven amante en lugar de pagar la escuela de su hija, lo que desemboca en que ambas acaben implicadas en una red de prostitución de lujo. Del mismo modo, el tráfico y el consumo de drogas está presente tanto en Baby como en Cómo vender drogas online (a toda pastilla) o Arenas movedizas, llegando a ser facilitadas por los propios progenitores en series de fantasía como Vampiros o The Innocents, para mantener ocultas las habilidades sobrenaturales de los personajes adolescentes incluso de sí mismos.

La sexualidad, eje fundamental sobre el que se desarrolla Sex Education, tiene una presencia relevante en el conjunto de ficciones analizadas, presentando en la mayoría de las ocasiones un grado de sofisticación impropio de las edades de los personajes, que en un $80,03 \%$ se corresponden con la franja comprendida entre los 16 y los 19 años. La representación de la diversidad de género va más allá de la aparición de personajes de distintas orientaciones, concretándose en escenas de sexo con más de dos participantes y la inclusión de términos como poliamor o pansexualidad, tratados con total naturalidad y demostrando un alto grado de madurez en las relaciones entre los miembros del grupo de pares, mostrando un entorno mayoritariamente comprensivo e integrador con respecto a los personajes de orientaciones distintas a la heteronormativa, si bien para los personajes homosexuales su condición sexual supone uno de los ejes fundamentales en torno al que se desarrollan tanto su personalidad como las acciones derivadas de la misma, destacando especialmente el caso de las ficciones italianas Baby y Suburra (aunque en esta última el personaje homosexual no entra dentro del análisis, estando en los primeros años de la veintena), donde parece existir una mayor presión social, hecho que puede considerarse como representación del localismo en estas producciones en un contexto, el italiano, en el que destaca incluso la falta de riqueza en el vocabulario mismo a la hora de referirse a la homosexualidad (Ranzato, 2012, p. 382). La única ficción en la que la homosexualidad de un personaje no resulta esencial para su desarrollo es en Ragnarok, donde Laurits Seier, hermano del protagonista, desvela al espectador su orientación en un comentario durante una cena familiar en el primer episodio, sin dar mayor importancia a la misma 
y no jugando, en ningún momento, un papel clave en las acciones llevadas a cabo por Laurits ni por su entorno. Este mismo personaje, al encontrarse con un grupo de estudiantes en el pasillo del instituto en el último episodio de la primera temporada, hace referencia con sorna a la representación de la diversidad racial en el mundo audiovisual publicitario, en una reflexión autoconsciente de la propia ficción hacia la aparente necesidad de cumplir con una cuota de representación, lo que enlaza, asimismo, con la homogenización del contexto audiovisual global. Además, esta diversidad racial conlleva para los propios personajes un desarrollo que, en ocasiones, se ve marcado por el color de su piel, como en el caso de Samir Said en Arenas movedizas o los hermanos Shanaa en Élite con el uso del hiyab para Nadia y la homosexualidad de Omar, que condicionan sus relaciones tanto con su grupo de pares como, especialmente, con su padre.

Otro aspecto a tener en cuenta en el desarrollo del personaje adolescente desde el punto de vista sociológico es la presencia del uso de las redes sociales (en aquellas ficciones en las que resulta posible, no apareciendo por razones obvias en tramas de carácter histórico o en series en las que el contexto en el que se desarrolla el relato hace inverosímil su presencia), que se posicionan como herramientas indispensables no ya para el establecimiento de relaciones entre personajes sino como clave fundamental en el desarrollo de las tramas. Las redes sociales conectan al personaje adolescente con su grupo de pares y con el mundo y las interacciones realizadas a través de estas tienen repercusiones en el mundo real (de la ficción). Incluidas en el relato audiovisual de forma que los mensajes se sobreimpresionan al plano del personaje que las está utilizando (como puede verse en Élite, Baby o Cómo vender drogas online (a toda pastilla) entre otras), la representación de estas se constituye como una referencia inequívoca al entorno multimediático y multipantallas en el que se encuentra inmerso el adolescente (personaje y espectador). Las redes sociales se constituyen, por un lado, como herramienta narrativa para el personaje y, por otro lado, como herramienta del fandom para el espectador en un contexto transnacional.

\section{Conclusiones}

Como plataforma de contenidos audiovisuales, Netflix se construye en torno a la idea de lo global (Lobato y Lotz, 2020, p. 133), tanto en términos de producción como, muy especialmente, de distribución. Con unas especificaciones técnicas determinadas para poder optar a pertenecer a su catálogo de originales, su sistema de distribución internacional es capaz de producir transformaciones en los distintos sistemas productivos locales, como en el caso español, donde la posibilidad de ampliar el target a un público global genera cambios en los procesos creativos nacionales, adaptando las ficciones a una duración determinada para adecuarse a las normas de circulación internacionales (Castro y Cascajosa, 2020). A través de la presente investigación se establece que este proceso de ajuste con respecto a la forma del producto audiovisual puede ser extrapolable al contenido, favoreciendo el desarrollo de un discurso transnacional en detrimento de las tramas locales. Con respecto a la representación adolescente en la ficción seriada original europea disponible en la plataforma, se observa, en primer lugar, que los personajes entre 13 y 19 años aparecen como parte del elenco regular en un $47,91 \%$ de las producciones ( 23 de las 48 series consideradas para el análisis); lo que no es óbice para que en el resto de ficciones también se encuentran 
presentes (por ejemplo, en las dos primeras temporadas de La casa de papel, emitidas por Antena 3 antes de que sus derechos de distribución fuesen adquiridos por Netflix, o en el tercer y último episodio de la miniserie Drácula, no pudiendo ser considerados por tanto personajes regulares). No encontrando distinciones en cuanto al protagonismo de esta tipología de personajes en los distintos países europeos cuyas ficciones conforman el corpus, se observa una media de entre 5 y 8,5 personajes adolescentes por serie, con España a la cabeza gracias a Élite.

Teniendo en cuenta las diferencias atribuibles tanto al género como a la época histórica representada en cada una de las 23 ficciones analizadas, la construcción de los personajes adolescentes presenta una serie de características comunes independientemente de la nacionalidad de sus series. De esta forma, se observa un predominio de personajes heterosexuales (con la inexistencia de identidades no normativas en la única serie para un público más infantil) y la utilización de la homosexualidad como elemento fundamental en el desarrollo de las tramas que atañen a estos personajes, así como conflictos derivados de la diversidad racial (tanto en Élite como en Baby, Suburra, Mortal, Arenas movedizas y, por supuesto, Kalifat), destacando como generador del conflicto no el color de la piel del personaje en sí, sino su situación económica, derivada del contexto que abocó a sus progenitores a migrar. La representación de la diversidad en las ficciones europeas se constituye como el reflejo de unas sociedades cada vez más globalizadas, destacando a este respecto que únicamente el 3,03\% de los personajes es extranjero en el contexto de la producción en la que aparecen, pero el $25,75 \%$ difiere de la mayoría caucásica. De esta forma, las series reflejan el resultado de los flujos migratorios de las pasadas décadas, presentando una serie de personajes adolescentes nacionales que representan a una generación de hijos de inmigrantes perfectamente integrada en el contexto sociocultural nacional.

Asimismo, si bien la diversidad de género refleja una posición de apertura con respecto a este tipo de identidades en el conjunto de sociedades europeas, especialmente a través de la representación de las generaciones más jóvenes, los comportamientos de los personajes adolescentes, fundamentalmente en lo relativo a cuestiones de índole sexual, muestran unos grados de sofisticación y complejidad que, en términos generales, los posiciona como jóvenes adultos que hacen gala de una experimentación impropia de su edad. Con una apariencia icónica mayoritariamente atractiva, los personajes femeninos destacan con un 66,66 \% frente al 58,06 \% de los masculinos, en un contexto en el que tanto dicha apariencia como la necesidad de conexión con el grupo de pares acerca las series europeas a la ficción original estadounidense de Netflix (Raya, Sánchez-Labella y Durán, 2018, pp. 139-140), quedando de esta forma corroboradas las hipótesis planteadas al inicio del presente artículo. La ficción seriada europea original de Netflix, que puede leerse como un gran discurso transnacional, refleja, a través de temáticas de actualidad social, el contexto sociocultural al que se enfrenta el adolescente europeo medio a través del uso de estereotipos que transcienden las fronteras nacionales en la creación de un arquetipo intercultural construido desde un punto de vista occidental.

Este hecho, reflejo de la sociedad globalizada, plantea una serie de cuestiones sobre las que merece la pena detenerse: por un lado, analizar si puede establecerse una relación similar entre las producciones europeas de los distintos medios locales no disponibles internacionalmente en plataformas de streaming; en segundo lugar, observar si el acceso al catálogo de originales de Netflix desde otros países arroja porcentajes similares a los percibidos desde España y si la tendencia homogeneizadora puede 
derivar en un mayor grado de similitud entre las ficciones de distintas nacionalidades y si es similar en otras plataformas de distribución de contenidos audiovisuales que operen de forma global; y, por último, teniendo en cuenta que del cómputo global de series originales accesibles desde España, segundo y tercer lugar lo ocupan producciones japonesas $(10,83 \%)$ y surcoreanas $(8,95 \%)$, analizar si puede establecerse una tendencia similar en las series asiáticas distribuidas transnacionalmente en Netflix.

\section{Bibliografía}

Ahn J. (2013). Samurai Champloo: Transnational Viewing. En E. Thompson y J. Mittell (eds.), How To Watch Television (pp. 364-372). New York: NYU Press.

Álvarez-Monzoncillo, J. M. (coord.). (2011). La televisión etiquetada. Nuevas audiencias, nuevos negocios. Barcelona/Madrid: Ariel/Fundación Telefónica.

Belmonte, J. y Guillamón, S. (2008). Coeducar la mirada contra los estereotipos de género en TV. Comunicar, 31, 115-120. doi:10.3916/c31-2008-01-014

Bermejo, J. (2012). Los personajes y las series de ficción en la vida de los y las jóvenes. Revista de Estudios de Juventud, 96, 31-49. Recuperado de

http://www.injuve.es/sites/default/files/Revista96_2.pdf

Border, E. (14 de abril de 2020). Spanish titles prove hit for Netflix. London: Ampere Analysis. Recuperado de https://www.ampereanalysis.com/insight/spanish-titles-prove-hit-for-netflix

Calvo González, S. y Saavedra Álvarez, C. (2020). "Por Trece Razones»: Trabajando educación sexual mediática con perspectiva de género en una teen serie. En L. Habib Mireles (coord.), Tecnología, diversidad e inclusión. Repensando el modelo educativo (pp. 54-63). Eindhoven/Madrid: Adaya Press.

Capapé, E. (2020). Nuevas formas de consumo de los contenidos televisivos en España: una revisión histórica (2006 - 2019). Estudios sobre el Mensaje Periodístico, 26 (2), 451-459. doi: $0.5209 /$ esmp.67733

Cascajosa Virino, C. (2016). La cultura de las series. Barcelona: Laertes.

Cascajosa Virino, C. (2018). De la televisión de pago al video bajo demanda. Análisis de la primera temporada de la estrategia de producción original de Movistar+. Fonseca, Journal of Communication, (17), 57-74. doi: 10.14201/fjc2018175774

Castro, D. y Cascajosa, C. (2020). From Netflix to Movistar+: How Subscription Video-on Demand Services Have Transformed Spanish TV Production. JCMS: Journal of Cinema and Media Studies, 59(3), 154-160. doi: 10.1353/cj.2020.0019

Casetti, F. y Di Chio, F. (2007). Cómo analizar un film. Barcelona: Ediciones Paidós.

Creeber, G. (ed.). (2004). The Television Genre Book. London: British Film Institute.

Davies, G. y Dikinson, K. (2004). Teen TV: Genre, Consumption \& Identity. London: BFI.

Fedele, M. y García-Muñoz, N. (2010). El consumo adolescente de la ficción seriada. Vivat Academia, 111, 47-64. doi: 10.15178/va.2010.111.47-64

Fernández-Ramírez, L. y McGowan, N. (2020). La programación de series internacionales en la televisión generalista española (1990 - 2010). Estudios sobre el Mensaje Periodístico, 26 (2), 541-553. doi: 10.5209/esmp.67686

Forni, D. (2020). Young adults and TV series. Netflix and new forms of serial narratives for young viewers. MeTis. Mondi educativi. Temi, indagini, suggestioni, 10 (1), 296-312. doi: 10.30557/MT00124

Galán Fajardo, E. (2006). Personajes, estereotipos y representaciones sociales. Una propuesta de estudio y análisis de la ficción televisiva. Revista ECO-PÓS, 9(1), 58-81. Recuperado de

https://e-archivo.uc3m.es/handle/10016/9475

García-Muñoz, N. y Fedele, M. (2011). Las series televisivas juveniles: tramas y conflictos en una «teen series». Comunicar, 37, 133-140. doi: 10.3916/C37-2011-03-05 
Graemer, T. (2019). Television Studies, We Need to Talk about «Binge-Viewing». Television \& New Media, 1-13. doi: 10.1177/1527476419877041

Greimas, A. J. (1987). Semántica estructural. Investigación metodológica. Madrid: Editorial Gredos.

Guarinos-Galán, V. (2009). Fenómenos televisivos 'teenagers': prototipias adolescentes en series vistas en España. Comunicar, 33, 203-211. doi: 10.3916/c33-2009-03-012

Herbert, D., Lotz, A. D. y Marshall, L. (2019). Approaching media industries comparatively: A case study of streaming. International Journal of Cultural Studies, 22 (3), 349-366. doi: $10.1177 / 1367877918813245$

Hoffner, C. y Buchanan, M. (2005). Young Adults Wishful Identification with Television Characters: The Role of Perceived Similarity and Character Attributes. Media Psychoogy, 7, 325-351. doi: 10.1207/S1532785XMEP0704_2

Jenkins, H. (2008). Convergence Culture: La cultura de la convergencia de los medios de comunicación. Barcelona: Paidós Ibérica.

Jenner, M. (2018). Netflix and the Re-invention of Television. Cambridge: Palgrave Macmillan.

Lacalle, C. (2015). Young people and television fiction. Reception analysis. Communications, 40 (2), 237-255. doi: 10.1515/commun-2015-0006

Lobato, R. y Lotz, A. D. (2020). Imagining Global Video: The Challenge of Netflix. JCMS: Journal of Cinema and Media Studies, 59(3), 132-136. doi: 10.1353/cj.2020.0034

Masanet, M.J., Medina-Bravo, P. y Ferrés, J. (2012). Representación mediática de la sexualidad en la ficción seriada dirigida a los jóvenes. Estudio de caso de Los Protegidos y Física o Química. Revista Comunicación, 10, 537-1548. Recuperado de

http://www.revistacomunicacion.org/pdf/n10/mesa9/117.Representacion_mediatica_de_la_ sexualidad_en_la_ficcion_seriada_dirigida_a_los_jovenes-Estudio_de_caso_de_Los_Protegidos_y_Fisica_O_Quimica.pdf

Medrano, C., Martínez de Morentín, J. I. y Apodaca, P. (2015). Perfiles de consumo televisivo: un estudio transcultural. Educación XX1, 18 (2), 305-321. doi: 10.5944/educxx1.14606

Meier, C. (2016). Studiocanal and the Changing Industrial Ladscape of European Cinema and Television. Media Industries, 3 (1), 49-63. Recuperado de

https://quod.lib.umich.edu/cgi/p/pod/dod-idx/studiocanal-and-the-changing-industrial-landscape.pdf?c=mij;idno=15031809.0003.104; format $=$ pdf

Mikos, L. (2019). Television as transitional medium. International Journal of Film and Media Arts, 4 (1), 6-13. doi: 10.24140/ijfma.v4.n1.01

Neale, S. (2004). Genre and Television. En G. Creeber (ed.), The Television Genre Book (pp. 3-4). London: British Film Institute.

Ojer, T. y Capapé, E. (2012). Nuevos modelos de negocio en la distribución de contenidos audiovisuales: el caso de Netflix. Revista Comunicación, 1 (10), 187-200. Recuperado de

http://www.revistacomunicacion.org/pdf/n10/mesa1/015.Nuevos_modelos_de_negocio_en_la_ distribucion_de_contenidos_audiovisuales-el_caso_de_Netflix.pdf

Propp, V. (2001). Morfología del cuento. Madrid: Ediciones Akal.

Ranzato, R. (2012). Gayspeak and Gay Subjects in Audiovisual Translation: Strategies in Italian Dubbing. Meta, 57 (2), 369-384. doi: 10.7202/1013951ar

Raya, I., Sánchez-Labella, I. y Durán, V. (2018). La construcción de los personajes protagonistas en las series de Netflix: el perfil del adolescente en 13 Reasons Why y en Atypical. Comunicación y Medios, 27 (37), 131-143. doi: 10.5354/0719-1529.2018.48631

Roel, M. (2020). Hacia una caracterización de los modelos de programación y audiencia de las televisiones generalistas «locomotora» en el umbral del apagón analógico (2005-2010): análisis del caso español. Estudios sobre el Mensaje Periodístico, 26 (2), 745-758. doi: 10.5209/ esmp.67439

Ross, S. M. y Stein, L. E. (eds.) (2008). Teen Television: Essays on Programming and Fandom. Jefferson: McFarland \& Company Publishers.

Savage, J. (2018). Teenage. La invención de la juventud 1875-1945. Madrid: Desperta Ferro Ediciones. 
